\title{
A User Guide to HyTech
}

\author{
Thomas A. Henzinger \\ Pei-Hsin Ho \\ Howard Wong-Toi
}

\begin{abstract}
HYTECH is a tool for the automated analysis of embedded systems. This document, designed for the first-time user of HYTECH, guides the reader through the underlying system model, and through the input language for describing and analyzing systems. The guide gives several examples of usage, and some hints for gaining maximal computational efficiency from the tool.

The version of HYTECH described in this guide was released in August 1995, and is available through anonymous ftp from ftp.cs.cornell.edu in the directory "pub/tah/HyTech, and through the World-Wide Web via HYTECH's home page http://www.cs.cornell.edu/Info/People/tah/hytech.html.
\end{abstract}

\section{Introduction}

The control of physical systems with embedded hardware and software is a growing application area for computerized systems. Since many embedded controllers occur in safety-critical situations, it is important to have reliable design methodologies that ensure that the controllers operate correctly. $\mathrm{HYTFCH}$ aids in the design of embedded systems by not only checking systems requirements, but also performing parametric analysis. Given a parametric system description, $\mathrm{HYTFCH}$ returns the exact conditions on the parameters for which the system satisfies its safety and timing requirements.

For completeness, we begin with a brief presentation of the underlying theoretical framework of linear hybrid automata [ $\mathrm{ACHH} 93, \mathrm{ACH}^{+} 95$ ], which we use to describe system specifications and requirement specifications. These automata model the continuous activities of analog variables (such as temperature, time, and distance), as well as discrete events (such as interrupts and output signals). Communication is modeled through event synchronization and shared variables. HYTECH's input consists of two

This research was supported in part by the ONR YIP award N00014-95-1-0520, by the NSF CARFFR award CCR-9501708, by the NSF grants CCR-9200794 and CCR9504469, by the AFOSR contract F49620-93-1-0056, and by the ARPA grant NAG2-892. 
parts: a system description and analysis commands. The system-description language allows us to represent linear hybrid automata textually. The tool forms the parallel composition of a collection of automata, each describing a modular component of an embedded system. The analysis-command language allows us to write simple iterative programs for performing tasks such as reachability analysis and error-trace generation.

We illustrate the use of the tool on several examples taken from the literature, and provide hints for a verification engineer to gain the maximal possible efficiency from HrTkch.

Outline Section 2 reviews linear hybrid automata, their semantics, parallel composition, and associated analysis techniques. A brief history of HYTFCH appears in Section 3. Sections 4 and 5 describe the HYTECH input language, first the system-description part, and then the analysis-command part. Section 6 illustrates the use of the tool on several examples. Section 7 is a short guide to designing specification requirements using HYTFCH's command language. Section 8 provides information on installing and running HYTFCH. Section 9 contains hints for the efficient use of HrTrch.

A full version of this user guide, including the complete grammar for the input language and additional examples, appears as [HHWT95b].

\section{Linear Hybrid Automata}

We model systems as the parallel composition of a collection of linear hybrid automata [ACHH93, $\mathrm{ACH}^{+}$95]. Informally, a linear hybrid automaton consists of a finite set $X$ of real-valued variables and a labeled multigraph. The vertices represent control modes, each with its own constraints on the slopes of variables in $X$. The edges represent discrete events and are labeled with guarded assignments to $X$. The state of the automaton changes either through the instantaneous action associated with an event or, while time elapses, through the continuous activity associated with a control mode. We also explicitly model urgent events, which must take place as soon as they are enabled (unless another instantaneous action disables them).

We use the linear hybrid automata that model a simple railroad crossing [LS85, AHH93] as a running example. The system consists of three components: a train, a gate, and a controller. The train is initially some distance - at least 2000 feet - away from the track intersection with the gate fully raised. As the train approaches, it triggers a sensor -1000 feet ahead of the intersection - signaling its upcoming entry to the controller. The controller then sends a lower command to the gate, after a delay of up to $\alpha$ seconds. When the gate receives a lower command, it lowers at a rate of 9 degrees per second. After the train has exited the intersection and is 100 feet away, it sends an exit signal to the controller. The controller then 


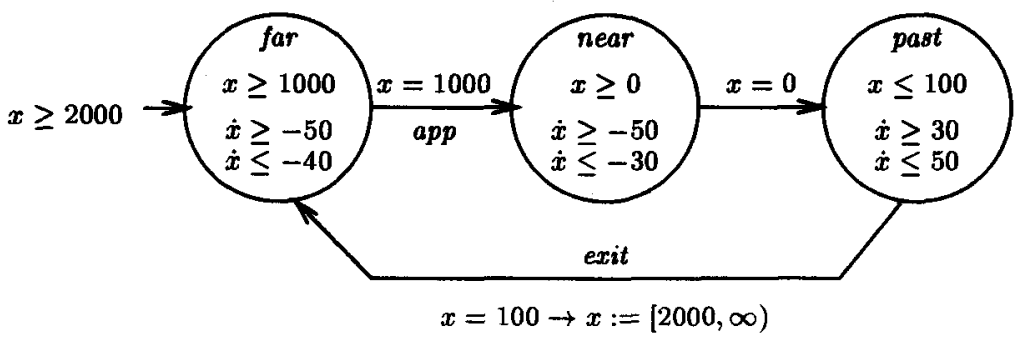

FIGURE 1. Train automaton

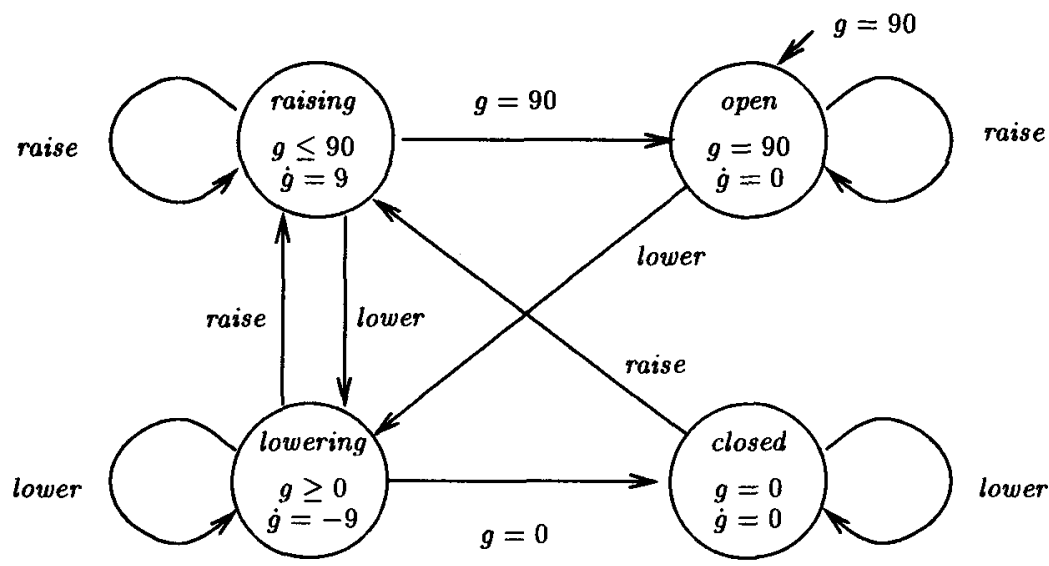

FIGURE 2. Gate automaton

commands the gate to be raised. The role of the controller is to ensure that the gate is always closed whenever the train is in the intersection, and that the gate is not closed unnecessarily long. The linear hybrid automata for the train, the gate, and the controller appear in Figures 1, 2 and 3.

\subsection{Definition}

We give an informal description of linear hybrid automata, and refer the reader to [AHH93, HHWT95a] for detailed definitions. A linear hybrid automaton consists of the following components.

Variables The automaton uses a finite ordered set $X=\left\{x_{1}, x_{2}, \ldots, x_{n}\right\}$ of real-valued variables to model continuous activities. For example, the position of the train is determined by the value of the variable $x$, which represents the distance of the train from the intersection. The variable $g$ models the angle of the gate. When $g=90$, the gate is completely open; 


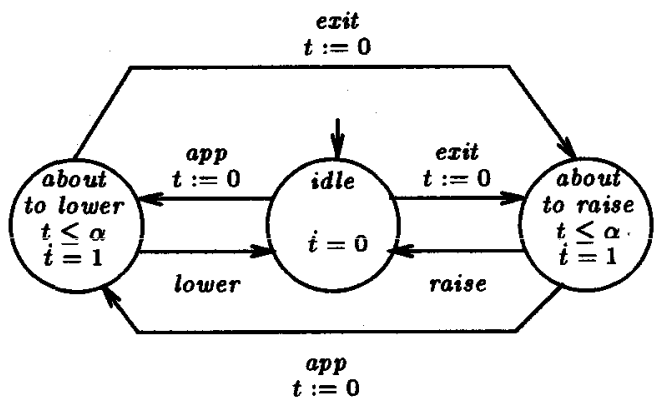

FIGURE 3. Controller automaton

when $g=0$, it is completely closed.

A valuation is a point $\left(a_{1}, a_{2}, \ldots, a_{n}\right)$ in the $n$-dimensional real space $\mathbb{R}^{n}$, or equivalently, a function that maps each variable $x_{i}$ to its value $a_{i}$. A linear expression over a set $X$ of variables is a linear combination of variables in $X$ with rational coefficients. A linear inequality is a non-strict ${ }^{1}$ inequality between linear expressions. A convex predicate is a finite conjunction of linear inequalities, e.g. $x_{1} \geq 3 \wedge 3 x_{2} \leq x_{3}+5 / 2$. A predicate is a finite disjunction of convex predicates, defining a set of valuations.

Locations Control modes are modeled using a finite set of vertices called locations. For example, the gate automaton has the locations open, raising, lowering, and closed. A state $(v, s)$ of the automaton $A$ consists of a location $v$ and a valuation $s$. We use the term region to refer to a set of states.

Initial condition There is a designated initial location and an initial predicate $\phi_{0}$ defining the set of initial values of the variables. For example, the gate is initially in location open with the value of $g$ equal to 90 . In the graphical representation, a small incoming arrow identifies the initial location, and is labeled with the predicate $\phi_{0}$.

Invariant conditions Each location $v$ is labeled with a convex predicate inv(v) over $X$, the invariant of $v$. The automaton control may reside in location $v$ only while its invariant is true, so the invariants can be used to enforce progress in the automaton. For example, in the gate automaton, inv $($ open $)=(g=90)$, inv (lowering) $=(g \geq 0)$, inv (raising $)=(g \leq 90)$, and $i n v($ closed $)=(g=0)$. The invariant at location lowering implies that the gate can only be lowered until it is fully closed, at which point control moves out to location closed. In the graphical representation, the invariant true is omitted.

\footnotetext{
'The requirement that all inequalities be non-strict is not essential. Our current implementation inherits this restriction from the polyhedral manipulation library we use.
} 
We are primarily interested in states $(v, s)$ where the valuation $s$ satisfies the location's invariant inv $(v)$. Such states are called admissible.

Transitions Discrete actions are modeled using edges between locations, which are called transitions. For example, the train automaton has three transitions; one from location far to location near for entering the region immediately surrounding the intersection, one from near to past for going through the intersection, and one from past to far for exiting the region around the intersection.

Each transition is labeled with a guarded command of the form $\phi \rightarrow \alpha$, where the guard $\phi$ is either the special predicate ASAP (which is always satisfied) or a convex predicate, and $\alpha$ is a set of assignments. Each assignment maps a variable into either a single linear expression over $X$, or a closed interval, whose endpoints are either finite (given as linear expressions over $X$ ), or infinite (given as $-\infty$ or $\infty$ ). In the train automaton, the transition between locations past and far is labeled with the guarded command $x=100 \rightarrow x:=[2000, \infty)$. In the graphical representation, we omit the guard true and empty assignment sets.

In order for a transition to take place from the state $(v, s)$ its guard must be satisfied in $s$. We describe how the set of assignments causes a change in the valuation from $s$ to some $s^{\prime}$. The lower and upper bound expressions of each assignment interval are evaluated at the valuation $s$, and each reassigned variable is nondeterministically given a value that lies in each interval to which it is assigned. If a variable cannot be assigned any value within the prescribed intervals, the transition cannot take place. Any variables for which there is no assignment in $\alpha$ remain unchanged. We define the binary transition-step relation, $\stackrel{\sigma}{\rightarrow}$, over admissible states such that $(v, s) \stackrel{\sigma}{\rightarrow}\left(v^{\prime}, s^{\prime}\right)$ iff the state $\left(v^{\prime}, s^{\prime}\right)$ can be reached from the state $(v, s)$ by taking a transition.

Each transition is optionally given a synchronization label. The synchronization labels are used to define the parallel composition of hybrid automata. For example, in the gate automaton, the transition from open to lowering has the synchronization label lower, and this synchronizes (i.e. must be taken simultaneously) with the transition labeled lower in the controller automaton.

A transition is urgent if its guard is AsAP. The full version [HHWT95b] of this guide illustrates the use of urgent transitions in the modeling of a distributed control system. There, a sensor waits to send a reading to the controller as soon as the controller is ready to receive the data.

Rate conditions We denote the rate of change of the variable $x \in X$ by $\dot{x}$, and we let $\dot{X}$ be the set $\left\{\dot{x}_{1}, \dot{x}_{2}, \ldots, \dot{x}_{n}\right\}$. Each control location $v$ is labeled with a convex predicate $\operatorname{act}(v)$ over $\dot{X}$, called the rate condition of $v$. For a given location, the rate condition restricts the rates of change of the variables. In the gate automaton, the rate condition for locations open and closed is $\dot{g}=0$, for location raising, it is $\dot{g}=9$, and for lowering, it is 
$\dot{g}=-9$. There is a technical restriction on the rate conditions allowed. All predicates that define bounded sets over $\dot{X}$ are permitted, and all examples in this guide meet this condition ${ }^{2}$.

A location $v$ is urgent if there is an urgent transition originating from $v$. No time is allowed to pass in such a location. We define the time-step relation, $\stackrel{\tau}{\rightarrow}$, such that $(v, s) \stackrel{\tau}{\rightarrow}\left(v^{\prime}, s^{\prime}\right)$ iff $v=v^{\prime}$, and there exists a real $\delta \geq 0$ such that $\delta>0$ implies $v$ is not urgent, and there is a function $f:[0, \delta] \rightarrow$ $\mathbb{R}^{n}$ such that (1) $f(0)=s,(2) f(\delta)=s^{\prime},(3)$ for all $t \in[0, \delta], f(t)$ satisfies $\operatorname{inv}(v)$, and (4) for all time $t \in(0, \delta)\left(d f_{1}(t) / d t, d f_{2}(t) / d t, \ldots, d f_{n}(t) / d t\right)$ satisfies $a c t(v)$, where $f_{i}(t)$ denotes the value of variable $x_{i}$ in the valuation $f(t)$.

\subsection{Parallel composition}

A hybrid system typically consists of several components which operate concurrently and communicate with each other. Each component is described as a separate linear hybrid automaton. The component automata coordinate through shared variables, and synchronization labels on the transitions are used to model message-type coordination. The linear hybrid automaton for the entire system is then obtained from the component automata using a product construction.

The control locations of the parallel composition of two automata $A_{1}$ and $A_{2}$ are pairs of locations, the first from $A_{1}$ and the second from $A_{2}$. The location $\left(v_{1}, v_{2}\right)$ has the conjunction of $v_{1}$ and $v_{2}$ 's invariants as its invariant, and the conjunction of their rate conditions as its rate condition. A location is initial iff its components are initial in their respective automata. The initial convex predicate is the conjunction of the components' initial convex predicates. Transitions from the components are interleaved, unless they share the same synchronization label, in which case they are synchronized and executed simultaneously, if at all. In the train-gate controller example, the system is composed of the train, gate, and controller automata of Figures 1,2 and 3. The controller communicates with the train by synchronizing on approach and exit events. It issues commands to the gate on the synchronized events raise and lower. The train's transition from location near to far is unlabeled, so it does not synchronize with any of the other components. In particular, this means the controller does not know the precise time at which the train enters the intersection.

We require a technical condition that the composition be well-formed: whenever two components synchronize on a label, if one transition has the guard ASAP then the other's guard must be either an ASAP guard or the

\footnotetext{
${ }^{2}$ The precise condition for the rate condition $\psi$ to be allowed is that the set of vectors $\{\dot{y} \mid$ there exists a real $k \geq 0$ and $\dot{x}$ satisfying $\psi$ such that $\dot{y}=k \dot{x}\}$ is bounded. In theory, the condition we require is not essential: it results from our current implementation's restriction to non-strict inequalities.
} 
predicate true (in which case the synchronized transition has guard ASAP), or the predicate false (in which case the synchronized transition has guard false).

\subsection{Reachability and safety verification}

At any time instant, the state of a hybrid automaton specifies a location and the values of all variables. If the automaton has the location set $V$ and $n$ variables, the state space is defined as $V \times \mathbb{R}^{n}$. We define the binary successor relation $\rightarrow$ A over states as $\stackrel{\tau}{\rightarrow} \cup \stackrel{\sigma}{\rightarrow}$. For a region $W$, we define $\operatorname{post}(W)$ to be the set of all successor states of $W$, i.e. all states reachable from a state in $W$ via a single transition or time step. The region forward reachable from $W$ is defined as the set of all states reachable from $W$ after a finite number of steps, i.e. the infinite union post ${ }^{*}(W)=\bigcup_{i>0}$ post $^{i}(W)$. Similarly, we define pre $(W)$ to be the set of all predecessor states of $W$, and we let the region backward reachable from $W$ be the infinite union $\operatorname{pre}^{*}(W)=\bigcup_{i>0} \operatorname{pre}^{i}(W)$.

In practice, many problems to be analyzed can be posed in a natural way as reachability problems. Often, the system is composed with a special monitor process that "watches" the system and enters a violation state whenever the execution violates a given safety requirement. Indeed all timed safety requirements [Hen92], including bounded-time response requirements, can be verified in this way. See Section 7 . A state $(v, s)$ is initial if $v$ is the initial location, and $s$ satisfies the initial predicate. A system with initial states $I$ is correct with respect to violation states $Y$ iff post $^{*}(I) \cap Y=\emptyset$, or equivalently iff $\operatorname{pre}^{*}(Y) \cap I$ is empty.

$\mathrm{HYTFCH}$ computes the forward reachable region by finding the limit of the infinite sequence $I, \operatorname{post}(I), \operatorname{post}^{2}(I), \ldots$ of regions. Analogously, the backward reachable region is found by iterating pre. These iteration schemes are semidecision procedures: there is no guarantee of termination. Nevertheless, we find that in practice, HYTFCH's reachability procedures terminate on most examples we have attempted. In addition, it has been shown that for a large class of systems [HKPV95], a linear hybrid automaton can be automatically preprocessed into an equivalent automaton over which the iterations converge.

\subsection{Parametric analysis}

A major strength of HYTFCH is its ability to perform parametric analysis. Often a system is described using parameters, and the system designer is interested in knowing which values of the parameters are required for correctness. Since the system is incorrect for parameter values for which there exists a state in the region $\operatorname{post}^{*}(I) \cap Y$, we may obtain necessary and sufficient conditions for system correctness by performing reachability analysis followed by existential quantification [CH78]. 
Our study of the train-gate controller demonstrates this technique. The controller decides when to issue lower commands to the gate based on the amount of time since the train last passed the sensor located 1000 feet ahead of the intersection. We consider the problem of determining exactly how long the controller can wait before issuing commands, while maintaining the requirement that the gate be closed whenever the train is within 10 feet of the intersection. The parameter $\alpha$ corresponds to the latest possible moment the controller can wait. We then use HYTF.CH to determine that the composed system includes violations whenever $\alpha$ is greater than or equal to $49 / 5$. Thus we conclude that the system is correct for values of the parameter strictly less than $49 / 5$.

\section{A Brief History of HYTECH}

\subsection{Implementation}

There have been three generations of HYTFCH. The very earliest prototype [AHH93] was written entirely in the symbolic computation tool Mathematica. Regions were represented as symbolic formulas. The evaluation of time-step successors used existential quantifications that are easily encoded in this language. While Mathematica offers powerful symbolic manipulation, and allows rapid development and experimentation with algorithms and heuristics, its operations over predicates turned out to be computationally inefficient. In particular, quantifier-elimination operations for computing time-step successors were expensive. HYTECH [HH95b] was rewritten to avoid this bottleneck in Mathematica. The second version of the verifier used a Mathematica main program that called efficient $\mathrm{C}^{++}$routines from Halbwachs' polyhedral manipulation library [Hal93, HRP94] for computing time-step successors. While this verifier achieved a total speed-up of roughly one order of magnitude, it required inefficient conversions between Mathematica expressions and $\mathrm{C}^{++}$data structures. It still relied on Mathematica for computing transition-step successors by substitution.

The third generation HYTFCH described here avoids Mathematica altogether and is built entirely in $\mathrm{C}^{++}$. It is roughly two to three orders of magnitude faster again than the second generation verifier. In addition, the input automata now allow nondeterministic assignments to variables, simultaneous assignments, more general rate conditions, and urgent events.

\subsection{A guide to HYTECH-related papers}

The following papers explain the theory behind linear hybrid automata in more detail, provide examples of their use, and discuss HYTFCH and related tools. 
Theory of hybrid automata Hybrid automata are based on timed automata [AD94] and were introduced in [ACHH93]. A related model appeared in the same volume [NOSY93]. Analysis methods included reachability and state-space minimization. The specification language Integrator Computation Tree Logic (ICTL) and a model-checking algorithm were introduced in [AHH93]. Approximations and abstract interpretation strategies for the algorithmic analysis of hybrid automata are discussed in the papers [HRP94, OSY94, HH95c]. The paper $\left[\mathrm{ACH}^{+} 95\right]$ provides an overview of the analysis techniques, including approximations. The analysis of nonlinear automata by translations to linear automata is described in [HH95a, HWT95a]. Decidability results appear in [Cer92, ACH93, KPSY93, AD94, MV94, PV94, BER94a, BER94b, BR95, MPS95, Hen95, HHK95, HKPV95]. In particular, [HKPV95] shows that the reachability problem is decidable, and HYTECH's analysis terminates, on the class of rectangular automata, where all convex predicates are of the form $a \leq x \leq b(a \leq \dot{x} \leq b)$.

HYTECH The earliest version of HYTFCH is mentioned in [AHH93], and performs full model-checking of ICTL formulas. The second generation of HYTF,C is discussed in [HH95b]. The thesis [Ho95] describes the first two generations of HYTECH in more detail, as well as summarizing much of the theory of hybrid automata. The current version of HYTECH is described in [HHWT95a]. The full version of this guide appears in [HHWT95b].

Case studies Numerous examples have been analyzed using linear hybrid automata. We mention only the first appearances of examples in the hybrid automata literature. A gas burner is studied in [ACHH93], together with a simple water monitor. The trajectories of a billiard ball, and the temperature of a reactor core are modeled in [NOSY93]. Fischer's timing-based mutual exclusion protocol is considered in [AHH93]. The paper [HH95b] includes a parametric analysis. A simple train-gate controller and a scheduler appear in [AHH93]. A manufacturing robot system and Corbett's distributed control system are also discussed in [HH95b]. The paper [HWT95b] describes the verification (see also [HH95b]) and error analysis of an audio control protocol. The benchmark generic railroad crossing example and an active structure controller are considered in [HHWT95a]. A nonlinear temperature controller appears in [HH95a], and a predator-prey system in [HWT95a].

Related Tools The analysis of linear hybrid automata supported by HYTFCH is based on symbolic region manipulation techniques first presented for real-time systems [HNSY94]. For the restricted case of real-time systems, these techniques have also been implemented in the tools KRONOS [NSY92, DOY94, $\mathrm{ACH}^{+}$95, DY95] and UPPAAI [LPY95]. Polka [Hal93, HRP94] is a tool for analyzing hybrid systems that concentrates on abstract interpretation strategies. 


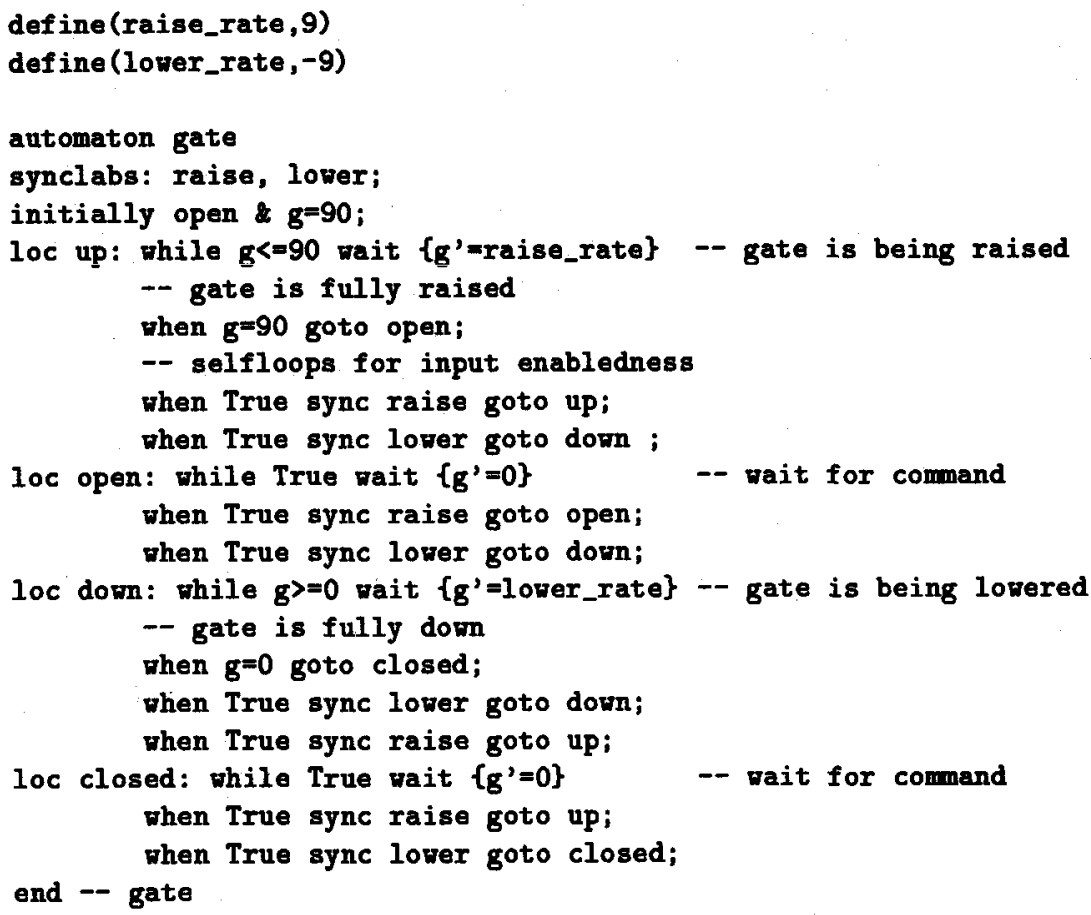

FIGURE 4. HYTECH input for the gate automaton

\section{Input Language: System Description}

HYTFCH's input consists of a text file containing a system description and a list of iterative analysis commands. The language is case-sensitive.

The system description language is a straightforward textual representation of linear hybrid automata. The user describes a system as the composition of a collection of components. Each component is given as a linear hybrid automaton. The system analyzed is taken as the product of all components given.

HrTech first passes its input through the macro preprocessor m4, allowing clear definition of constants in the system ${ }^{3}$. For example, we may declare and use the constant raise_rate in the gate automaton of Figure 2, as shown in the sample HYTFCH input appearing in Figure 4. Whitespace (blank spaces, tabs, new lines) between tokens is ignored. The syntax is described in more detail below. The complete grammar appears in [HHWT95b].

Comments The rest of an input line after two adjacent dashes (--) is taken as a comment.

\footnotetext{
${ }^{3}$ For details of the Unix command m4, type man $m 4$.
} 
Variables All variables in the system are declared at the top of the description, in a single declaration. Variables may be of the following types: discrete, clock, stopwatch, parameter, analog. The type declarations allow more readable descriptions and enable simple static checking by the parser. A clock variable always has rate 1 , and a discrete variable always has rate 0 . The rate of a stopwatch must be either 0 or 1 . Parameters have rate 0 in all locations, and may never be assigned values. Analog variables have no syntactic restrictions. Variables of type discrete, clock and parameter are said to be fixed rate variables, since their rate intervals are fixed by their type, namely 0,1 and 0 respectively. Constraints on their rates are automatically added to the rate conditions for each location; indeed, it is illegal for the user to constrain explicitly the rate of a fixed rate variable. For example, the variables for the train-gate controller example are declared as

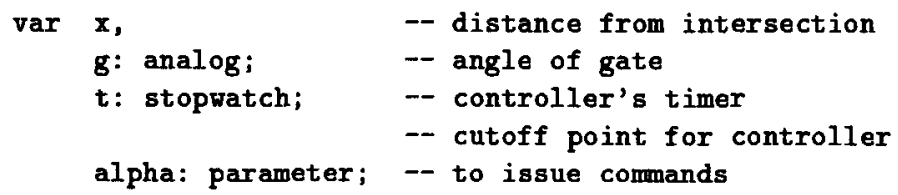

Linear terms, expressions and constraints A linear term is either (a) a variable multiplied by a rational coefficient, or (b) a rational number. A linear expression is an additive combination of linear terms. A linear constraint is a non-strict inequality $(<=,>=)$ or equality $(=)$ between linear expressions. Note that rational coefficients must either (a) be an integer, (b) have an integer as numerator and a nonzero integer as denominator, or (c) be omitted, in which case it is understood to be 1 . For example, 1/2x $-24 / 5 y<=z+5 t-6+y$ is a syntactically legal linear constraint.

Automaton components Each automaton is given a name which may be used later in the specification. Its synchronization labels are declared. Its initial location and the initial condition on its variables must also be provided. For example, the header for the train automaton is as follows:

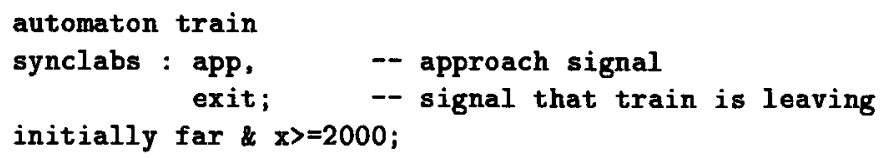

Each automaton component includes a list of locations, described below, terminated by the keyword end.

Locations Each location is named and labeled with its invariant. Rate conditions may also be provided. The syntax $g$ ' in $[10,20]$ is shorthand for $\left.g^{\prime}\right\rangle=10 \& g^{\prime}\langle=20$. For example, loc far: while $x\rangle=100$ wait $\{x$ in $[-50,-40]\}$ is the header for the location far with invariant $x \geq$ 100 , and rate condition $-50 \leq \dot{x} \leq-40$. 
Invariants may be conjunctions of linear constraints, such as $x>=1 / 2$ \& $y<=2 / 3+x$, but must not be disjunctions ${ }^{4}$. Conjunctive rate conditions are separated by commas, as in wait $\left\{x^{\prime}=z^{\prime}, y^{\prime}\right.$ in $\left.[2,4]\right\}$.

Each location is associated with a list of transitions originating from it.

Transitions Each transition lists a guard on enablement and the successor location. Both the synchronization label and the assignments are optional. Infinite bounds are expressed as either-inf or inf. For example, the following are legal transitions.

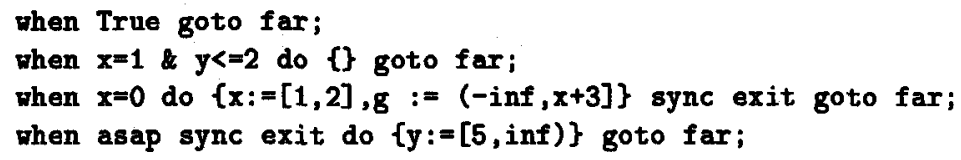

Again, notice that guards may be conjunctions of linear constraints, but not disjunctions (use multiple transitions). Also, the order of the synchronization information and the assignments is interchangeable, if they appear at all, but the guard must appear first and the successor location last. The ASAP guard on the last transition listed indicates it is an urgent transition which must take place as soon as possible. Recall that there is a syntactic restriction that non-trivial guards are not permitted on urgent transitions or any transitions in other components with the same synchronization label as an urgent transition.

Composition It is assumed that the system being described is the parallel composition of all listed components.

\section{Input Language: Analysis Commands}

The analysis section of the input consists of two parts: declaration of variables for regions, and a sequence of iterative command statements. Analysis commands provide a means of manipulating and outputting regions. Commands are built using objects of two basic types: region expressions for describing regions of interest, and boolean expressions used in the control of command statements. Regions may be stored in variables, provided the region variables are declared via a statement such as

var

init_reg, final_reg: region;

which declares two region variables called init_reg and final_reg. HҮTFCH provides a number of operations for manipulating regions, including computing the reachable set, successor operations, existential quantification, convex hull, and basic boolean operations.

\footnotetext{
${ }^{4}$ In order to model a disjunctive invariant, split the location into several locations, one for each disjunct [AHH93].
} 


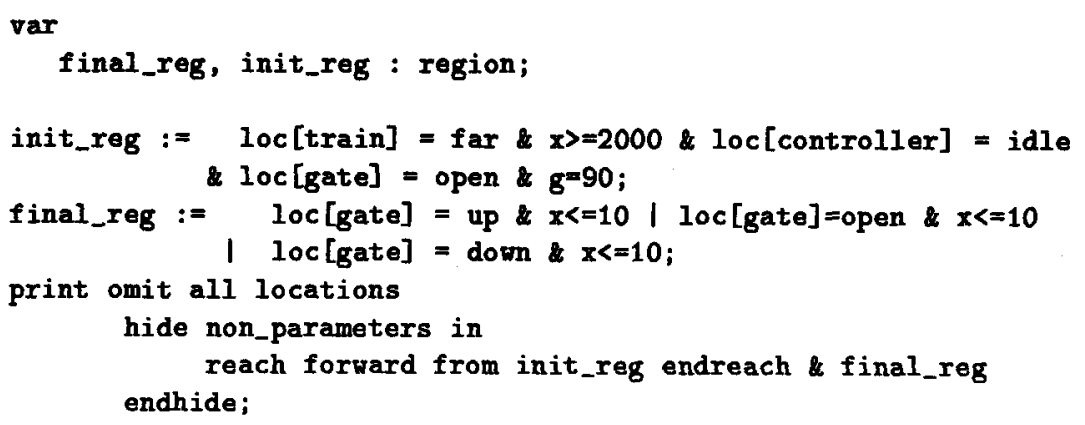

FIGURE 5. Analysis commands for train-gate controller

For example, the specification commands in Figure 5 are for analyzing the train-gate controller. Their overall effect is to determine the critical bound on the parameter $\alpha$. First, the two regions final_reg and init_reg are declared. The first two statements assign values to these regions using direct constraints on the states. Notice that disjunctions may be used. The third statement outputs the constraint on the parameter $\alpha$ under which the system is not correct. This printing command is given by the prefix print omit all locations, which tells HrTwCH to output the region enclosed between the words hide and endhide, but only after hiding all information about locations. We choose to omit all location information since for any particular value of $\alpha$ the specific final location reached is irrelevant. HYTFCH evaluates the region expression between the hide keywords by first performing reachability analysis from the initial region specified by init_reg, intersecting the reachable states with the final region (final_reg), and then existentially quantifying out all variables that are not declared as parameters. After 1.72 seconds computation on a Sparcstation 20, $\mathrm{HrTFCH}$ produces the following output, showing that the system is correct whenever $\alpha<49 / 5$.

5alpha $>=49$

\subsection{Region expressions}

Region expressions are built from linear inequalities, constraints on locations, and region names, by existential quantification, pre, post, and convex hull operations, reachability, conjunction, and disjunction. Each region expression defines a region. The symbol 〈reg_exp $\rangle$ denotes an arbitrary region expression.

Linear inequalities The most basic region expression is a linear inequality. For example, $\mathrm{x}<=100$ is a region expression, defining the set of all states where the variable $x$ has value no greater than 100 .

Location constraints $10 \mathrm{c}[\langle$ aut_name $\rangle]=($ loc_name $\rangle$.

The location name (loc_name) must be the name of a location in the 
automaton $\left\langle a u t \_n a m e\right\rangle$. For example, the region expression loc [gate] $=$ open defines the set of all states where the location component corresponding to the gate is open.

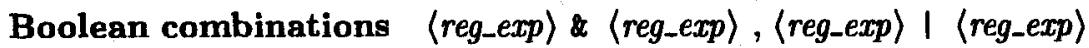
The disjunction of region expressions, written using the operator $I$, is a region expression (representing the union of its operands), as is the conjunction of region expressions (representing the intersection of its operands), written with the operator \&. The \& operator has precedence, so that an expression without parentheses is considered to be a disjunction of conjunctions. In addition, the boolean constants True and False have the expected meaning.

Parentheses Expressions not in conjunctive normal form may be given using parentheses. For example, $x<=4 \&(y<=5 \mid y>=5)$ is equivalent to $x<=4$.

Region name A region expression may be any declared region variable. There is no automatic check that the region variable has been assigned a value. The value of the expression is the region most recently assigned to the variable.

Existential quantification hide $\left\langle v a r_{-}\right.$list $\rangle$in $\left\langle r_{\text {reg_exp }}\right\rangle$ endhide The hide expression evaluates to the region obtained by existentially quantifying a list of variables. For example, the command print hide $x$ in $x<=1 \& x=y$ endhide outputs the region where $y \leq 1$. In general, quantified variables may be listed, separated by commas, as in print hide $x, z$ in $x<=1 \& y<=x+3 \& z=y-x$ endhide. Alternatively, the list $\langle$ var_list $\rangle$ may be replaced by the keywords all (for all variables) or non_parameters (for all variables not declared as parameters).

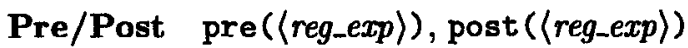

The pre and post expressions evaluate to the regions obtained by applying pre and post respectively to their arguments.

Convex hull hull $(\langle$ reg_exp $\rangle)$

The expression hull ( $\langle$ reg_exp $\rangle)$ returns the region where each location $v$ is associated with the convex hull of all valuations $s$ for which $(v, s)$ is in the region defined by $\langle$ reg_exp $\rangle$. For example,

$$
\begin{aligned}
& \operatorname{loc} 1:=\operatorname{loc}[\mathrm{P} 1]=10 c_{-} \text {a \& loc }[\mathrm{P} 2]=10 c_{-} \mathrm{b}_{-} 1 \text {; } \\
& \text { loc2 := loc }[\mathrm{P} 1]=10 c_{-} a \text { \& } \operatorname{loc}[\mathrm{P} 2]=\operatorname{loc}_{-} \mathrm{b}_{-} 2 \text {; } \\
& \text { approx := hull (10c1 \& } x=0 \mid \text { loc1 \& } x=1 \mid \text { loc2 \& } x=1) \text {; }
\end{aligned}
$$

assigns approx the region represented by 1 oc $180<=x \& x<=1$ | loc $2 \& x=1$.

$$
\begin{aligned}
\text { Reachability } & \text { reach forward from }\langle\text { reg_exp }\rangle \text { endreach } \\
& \text { reach backward from }\langle\text { reg_exp } \text { endreach }
\end{aligned}
$$

There are two specialized expressions for returning the set of states reachable from any arbitrary region: one for forward reachability and one for 
backward reachability. For example, the expression reach forward from init reg endreach appearing in the analysis commands in Figure 5 evaluates to the region reachable from init_reg by iterating post. The backward reachability expression iterates pre until convergence.

\subsection{Boolean expressions}

Boolean expressions are built from region comparisons and region emptiness checks using boolean operators. Boolean expressions are used in conditional statements and while loops. The symbol (bool_exp) denotes an arbitrary boolean expression.

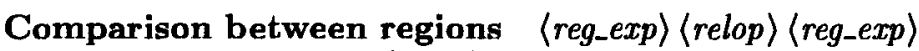

The relational operator $\langle$ relop $\rangle$ is one of the symbols $<,<=,=,\rangle=$, and $>$, representing the binary set comparison operators $C, \subseteq,=, \supseteq$, and $\supset$ respectively. For example, the following are legal boolean expressions.

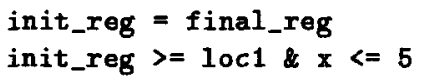

Emptiness empty $\left(\left\langle\right.\right.$ reg_exp $\left._{\rangle}\right)$

The unary predicate empty applied to a region expression evaluates to true iff its argument contains no states. For example, the following code could be used to determine whether the system satisfies its safety requirement.

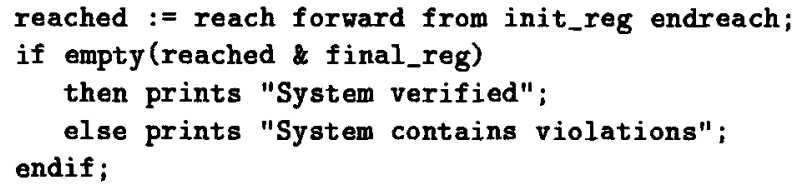

Boolean combinations $\langle$ bool_exp $\rangle$ and $\left\langle b o o l_{-} e x p\right\rangle,\left\langle b o o l_{-} e x p\right\rangle$ or $\left\langle b o o l \_e x p\right\rangle$ not 〈bool_exp〉

Boolean expressions may be combined to yield boolean expressions. The negation of a boolean expression is a boolean expression. For example, not empty (reached) is a boolean expression. The conjunction and disjunction of boolean expressions is a boolean expression, with the natural meaning, written using the keywords and and or. Note that region expressions use the symbols \& and 1. Negation has highest priority and conjunctions bind more tightly than disjunctions.

\subsection{Command statements}

There are commands to perform common tasks such as error-trace generation and parametric analysis. Command statements are built from primitives for printing and assigning regions. Command statements may also occur within conditional statements and while statements. Each command is terminated by a semicolon. 
Printing There are four basic commands for outputting information. All output appears on stdout.

print $\langle$ reg_exp $\rangle$ The basic print command outputs the states in the region defined by its region expression argument. For example, the command print init_reg (see Figure 5) would produce the output

Location: far.idle.open

$g=90 \& x>=2000$

The valuations associated with a location $v$ within a region $W$ are the valuations $s$ such that $(v, s) \in W$. The print command prints out a list of locations and predicates defining the states associated with them. Non-convex predicates are output as disjunctions of convex predicates. Locations for which there are no associated valuations in the region do not appear in the output. The string far.idle.open indicates that the valuations satisfying the convex predicate $g=90 \wedge$ $x \geq 2000$ are associated with the control location where the train component is far from the intersection, the controller component is in its idle location, and the gate component in its open location. Note that location information is printed with periods separating the locations for each component, and that components are listed in the order in which they are declared.

print omit 〈loc_list) locations 〈reg_exp This command generalizes the basic print command by first eliminating information about the locations of all components listed after the omit keyword. For example, if strange_reg is first assigned to

init_reg | loc[gate]=closed \& $1000<=x \&$ loc[train] $=$ far

then print omit gate, controller locations strangereg produces the output

Location: far.

$x>=1000$

indicating that the region given includes only locations in the product automaton for which the train component is in its far location, and that all valuations for which the value of $x$ is greater than or equal to 1000 appear in some such location. The absence of a location name for the second and third component automata indicates that information for these components' locations has been existentially quantified.

As shorthand, the keyword all may appear in place of a list of automata names, in which case all location information is quantified out, as in Figure 5. 
prints (string) This command prints strings, enclosed in double quotes, directly to stdout. For example, the statement prints "Hi there" outputs the string "Hi there" followed by a carriage return.

printsize (reg_name) This command prints information about the "size" of the region stored in the region variable given as an argument. Information output includes the number of product locations for which the associated predicate is nonempty and the total number of convex predicates used in representing the region.

Assignment $\langle$ reg_name $\rangle:=\langle$ reg_exp $\rangle$

Any region expression may be assigned to any region name. For example, we may initialize the final region with the statement

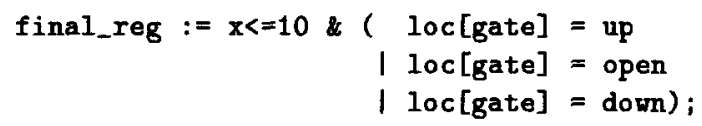

which is equivalent to the assignment appearing in Figure 5.

Conditional The if-then and if-then-else statements have the expected meaning. For example, the following are legal conditional statements.

if init_reg<=final_reg then prints "Hi"; print strange_reg; endif;

if init_reg=final_reg then prints "Equal";

else prints "Not equal"; endif;

The boolean expression comparing regions is first evaluated, and then the appropriate list of statements (if any) is executed.

Iteration The while statement has the expected meaning. For example,

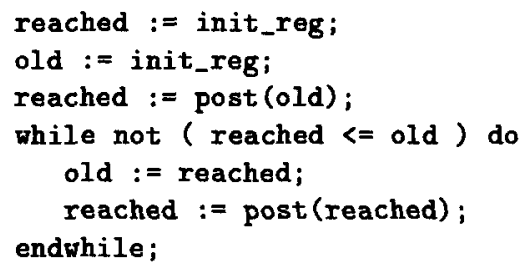

computes the set of reachable states from the initial states by iterating the post operation until a fixpoint is obtained.

Error trace generation print trace to 〈reg_exp using 〈reg_name HYTFCH provides a simple facility for generating error traces for faulty systems. One must first use the built-in reachability utility (see Subsection 5.1), which causes $\mathrm{HYTFCH}$ to store internal information that can be used to generate traces. Second, the command to generate traces is issued, specifying both the target region of the traces, and the name of the region variable previously used to store the result of the reachability analysis. This is best illustrated by an example. Suppose we are using forward reachability 


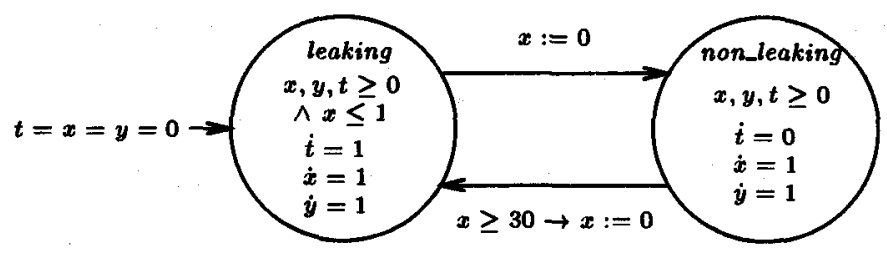

FIGURE 6. Automaton for the leaking gas burner

analysis to see whether any state in the violation region final_reg is reachable from the initial region init_reg. The following sequence of commands causes $\mathrm{HYTFCH}$ to generate an error trace, if one exists.

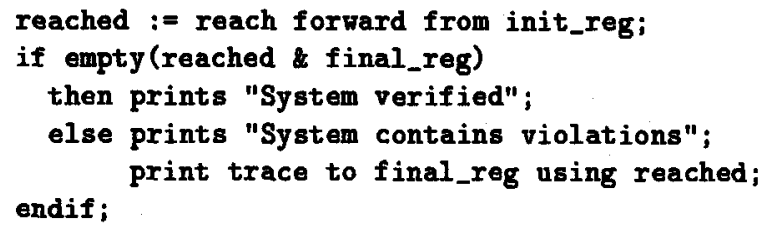

The trace output consists of regions, i.e. sets of states, not individual states. Each region will be accessible from the previous via a time step allowing the continuous variables to evolve, followed by a transition step. The trace generated is minimal in length, and includes the synchronization labels, if any, for transitions between regions along the trace. Regardless of whether forward or backward reachability is used, the trace is always printed in an absolute forward direction.

Note: this command is rather fragile, and should be used with some care. The error trace generation command always assumes - without any automatic checks - that the region variable appearing after the keyword using (reached in the above example) has been assigned a reachable region using the built-in reach expression, and that no reach expression has since been evaluated.

\section{Examples}

Additional examples may be found in the directory examples of the software distribution. We discuss two of them here in more detail.

\subsection{Gas burner}

The "leaking gas burner" example has appeared in the early literature on formal methods applied to hybrid systems [CHR.91, ACHH93]. We show how this simple system can be analyzed in HYTFCH. The gas burner is 


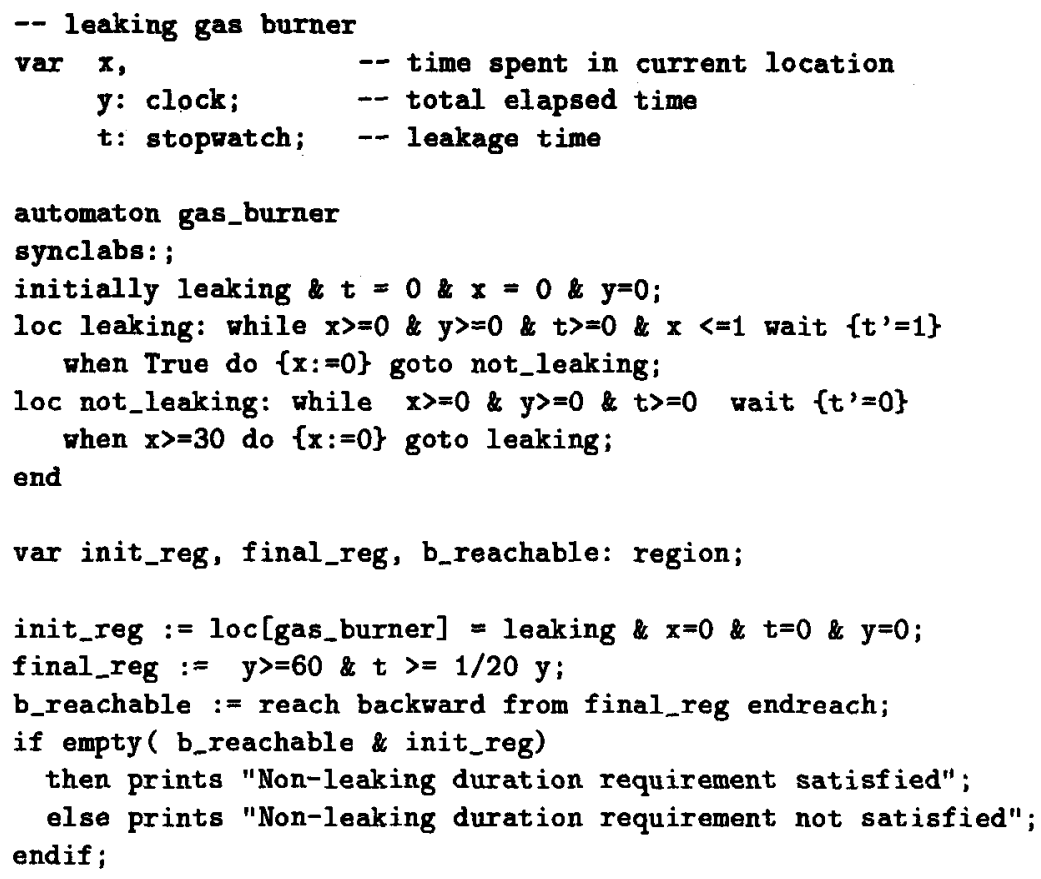

FIGURE 7. Input file for the analysis of the gas burner

in one of two modes; it is either leaking or not leaking. Leakages are detected and stopped within 1 second. Furthermore, once a leakage has been stopped, the burner is guaranteed not to leak again until at least 30 seconds later. The system is initially leaking.

The linear hybrid automaton of Figure 6 models the gas burner. The clock $x$ records the time elapsed since last entering the current location, and is sufficient for modeling the behavior of the system. However, in order to analyze the system, we need to add the auxiliary variables $t$ and $y$. The stopwatch $t$ measures the cumulative leakage time. It increases at rate 1 in the location leaking, and at rate 0 in location non_leaking. The clock $y$ measures the total elapsed time. Using these auxiliary variables, we prove that if at least 60 seconds have passed, then the burner has been leaking for less than one twentieth of the total elapsed time. The requirement holds unless there is a state, forward reachable from the initial states, in which $y \geq 60$ and $t \geq y / 20$. We compute the region backward reachable from all states satisfying $y \geq 60 \wedge t \geq y / 20$. Since this region does not include any initial states, the requirement is satisfied. In fact, forward reachability for this system does not terminate. In general, it is not easy to determine ahead of time whether forward or backward reachability analysis is preferable.

The complete input file for this example appears in Figure 7. HYTFCH outputs the string "Non-leaking duration requirement satisfied". The com- 

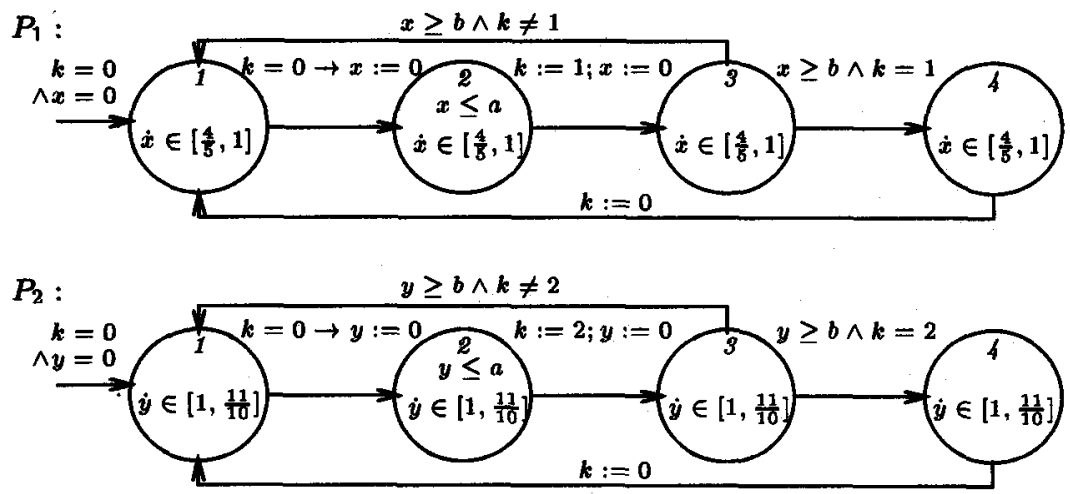

FIGURE 8. Automata for processes $P_{1}$ and $P_{2}$ in Fischer's mutual exclusion protocol

putation takes 0.62 seconds on a SparcStation 20, using a maximum of 0.73 MB of memory.

\subsection{Fischer's mutual exclusion protocol}

We demonstrate parametric analysis through a drifting clock version of the simple timing-based mutual-exclusion protocol due to Fischer [Lam87, AHH93]. The system consists of two processes, $P_{1}$ and $P_{2}$, each performing atomic read and write operations on a shared memory variable $k$. Each process $P_{i}$, for $i=1,2$, models the following algorithm:

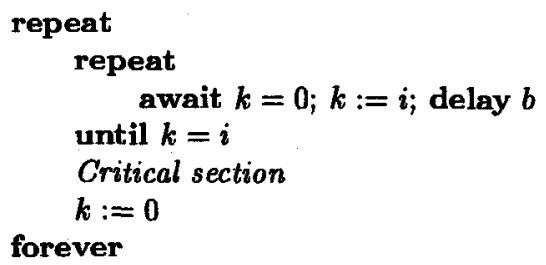

The instruction delay $b$ delays a process for at least $b$ time units as measured by its local clock. Each process uses its own local clock to measure the delay times. Process $P_{i}$ is allowed to enter its critical section iff $k=i$. Furthermore, each process takes no more than $a$ local time units to write a value into the variable $k$, i.e. the assignment $k:=i$ occurs within $a$ time units after the await statement completes. To complicate matters, the two processes use drifting clocks. Process $P_{1}$ 's clock is slow, and its rate may vary between 0.8 and 1 , while that of $P_{2}$ is fast with rate between 1 and 1.1 .

The automata for the two processes appear in Figure 8. Each process is modeled using the private clocks $x$ and $y$, respectively. Each process has 
a critical section, represented by the location 4 in each automaton. The invariants at location 2 ensure the upper time bound on the write access to $k$, while the guards on the transitions from location 3 to location 4 model the lower time bound of the delay.

We perform parametric analysis to determine the values for $a$ and $b$, if any, for which mutual exclusion holds. The "unsafe" region is characterized by the region expression $10 c[P 1]=10 c \_4$ \& $10 c[P 2]=10 c_{-} 4$. As for the train-gate controller example, we are interested in the values of the parameters for which there exists a reachable unsafe state. These values are output using the print omit all locations analysis command, in conjunction with existential quantification of the non-parameter variables:

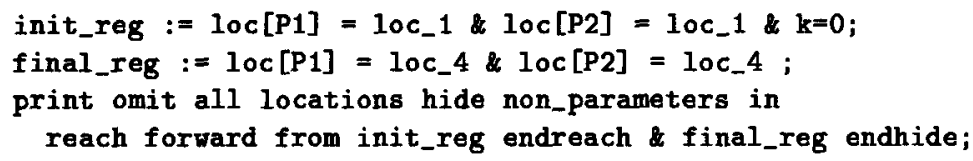

HYTF,CH's computation takes 3.79 seconds using $1.1 \mathrm{MB}$ of memory, producing the following output, which indicates that the system is correct whenever $a<8 b / 11$.

$11 \mathrm{a}>=8 \mathrm{~b} \& \mathrm{a}>=0$

\section{Designing Requirement Specifications}

It is not always obvious how to specify requirements of systems. This section provides some hints to the verification engineer by outlining how to check for many common classes of requirements. All forms of specifications below rely on the use of reachability analysis.

\subsection{Simple safety}

A safety requirement intuitively asserts that "nothing bad ever happens". Many specifications are expressed naturally as safety requirements. A system is said to be correct iff its reachable states all satisfy an invariant $\phi$, defining a set of safe states: the "bad thing" to happen is to reach a state that does not satisfy the invariant ${ }^{5}$. For example, Fischer's mutual exclusion protocol should guarantee that processes $P_{1}$ and $P_{2}$ are never in their critical sections at the same time. Also, the train-gate controller is required to ensure that the gate is always down whenever the train is within 10 feet.

As discussed above (Subsection 2.3), safety requirements can be verified in HYTFCH using the region $\neg \phi$. One method is to perform forward reachability analysis from the system's initial states, and then check whether

\footnotetext{
${ }^{5}$ The reader familiar with temporal logics should observe that such requirements are expressed in the form $\forall \square \phi$, meaning intuitively that $\phi$ is always true for all reachable states of the system.
} 
the intersection with the violating states $\neg \phi$ is empty. Assuming the region init_reg has been assigned the set of initial states, and viol has been set to the region $\neg \phi$, the following HҮTЕсн input checks the safety requirement, and generates an error trace if any exist.

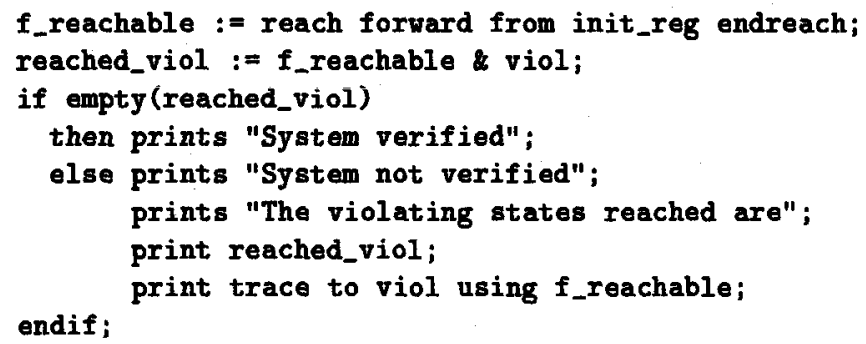

Alternatively, the analogous backward reachability analysis can be used.

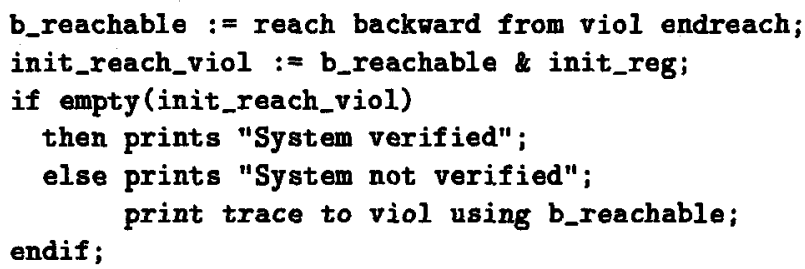

Strict equalities When the invariant $\phi$ involves non-strict inequalities, it may be impossible to express the violating states $\neg \phi$ using only nonstrict inequalities. This problem can be overcome in two different ways. First, if the invariant $\phi$ itself can be expressed using non-strict inequalities only, HYTFCH can check directly whether all reachable states satisfy the invariant using the containment operator.

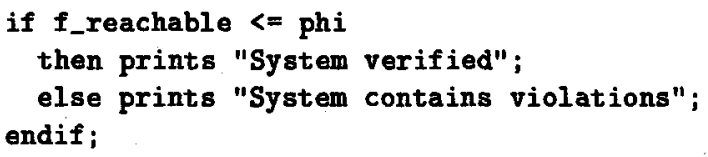

Alternatively, one may instead use the set closure $(\neg \phi)^{c}$ of $\neg \phi$ as the set of violating states, and then check that the only reachable states in $(\neg \phi)^{c}$ lie in $\phi$, or equivalently, lie in the intersection of $(\neg \phi)^{c}$ and $\phi$. For example, consider the task of verifying the gate is always down whenever the train is strictly less than 10 feet away. The invariant $\phi$ is given as loc [gate] $=$ down $\vee x \geq 10$. Its negation $\neg \phi$ is $\operatorname{loc}[$ gate $] \neq$ down $\wedge x<10$, which is inexpressible using non-strict inequalities only. The following analysis can be used to verify this requirement.

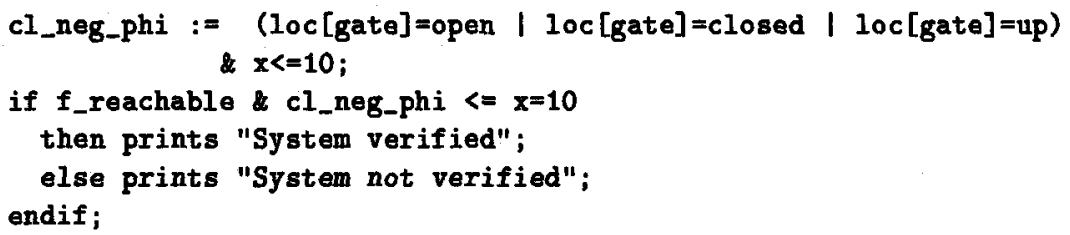


Despite being more complicated, this alternative is often faster than the first, since the $<=$ operator can be expensive when applied to complex expressions.

\subsection{Simple possibility}

A simple possibility requirement asserts that "something good can always happen." If the notion of "something good" can be expressed as a region expression $\phi$, then such requirements maintain that all states forward reachable from the initial states are backward reachable from a state in $\phi .{ }^{6}$ For example, we may wish to prove that for Fischer's mutual exclusion protocol, there is always a possibility that process $P_{1}$ will enter its critical section sometime in the future. The following HYTFCH code checks this assertion.

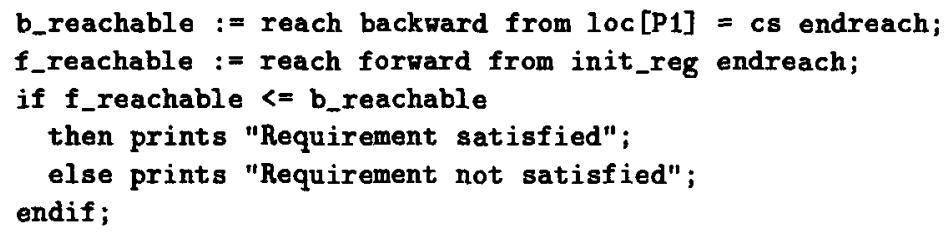

\subsection{Simple real-time and duration requirements}

Many simple real-time requirements can be specified by introducing clocks and stopwatches to measure delays between events, or the length of time a particular condition holds. In the gas burner example, we assert that as long as a minute or more has passed, the burner has been leaking no more than $5 \%$ of the time. In this case, we introduce a new variable for each time duration of interest. We need to know the total elapsed time and the time spent in location leaking. These quantities are measured by the clock $y$ and stopwatch $t$ respectively. The duration requirement we are interested in then becomes the safety requirement where the violating states are given by the predicate $y \geq 60 \wedge t \geq y / 20$.

\subsection{Additional requirements}

By no means do all requirement specifications fall into the categories discussed above. However, a simple technique can be used to reduce many requirements to safety requirements. The idea is to build a separate monitor automaton for the requirement being checked [VW86]. The monitor typically contains special states which are only reachable by violating executions. The monitor must act strictly as an observer of the original system, without changing its behavior. Reachability analysis may then be performed on the parallel composition of the system and the monitor, with the

\footnotetext{
${ }^{6}$ These requirements are expressed in temporal logics in the form $\forall \square \exists \diamond \phi$.
} 


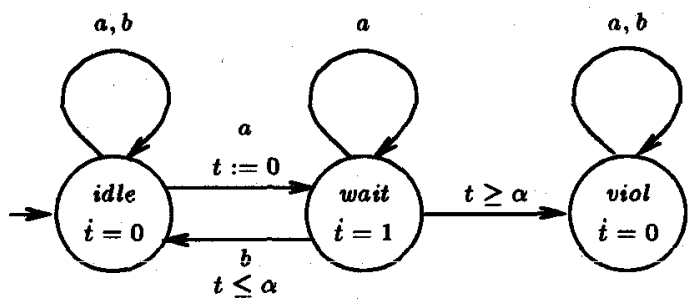

FIGURE 9. Generic bounded-response monitor automaton

system correct iff no violating state in the monitor is reached. To illustrate the technique, we use the category of bounded response requirements.

Bounded response $A$ bounded-response requirement asserts that if something (a trigger event, $a$ say) happens, then a response, $b$ say, occurs strictly within a certain time limit $\alpha{ }^{7}$ For example, one may assert that every approach of the train is followed by a raise command within 10 seconds. To verify these requirements, it is often easiest to introduce a new stopwatch variable, $t$ say, and build a monitor process with three locations: idle, wait and viol. Figure 9 depicts a generic automaton for bounded response requirements. Control is initially in the idle location. When a trigger event occurs in a non-violating location, control may pass to the wait location and the clock $t$ is reset. Response events cause control to return to the idle location. The unlabeled transition from the wait location to viol is only enabled when $t \geq \alpha$, i.e. time for the response event has passed by. This automaton will reach its violation location iff it is possible for $\alpha$ time units to pass after an $a$ event without $a b$ event occurring. Therefore, the violation location is not reachable iff every $a$ event is followed by a $b$ event occurring less than $\alpha$ time units later.

To assert that the response event may occur any time up to and including $\alpha$ time units after the trigger event, we may use the same monitor automaton as above, but checking that the violation location is only ever reached with the value of $t$ being $\alpha$.

Since bounded response requirements occur frequently, we demonstrate how strict bounded response requirements can be verified slightly more efficiently, i.e. the response event must occur before the response timeoccurring when exactly $\alpha$ time units have passed is not acceptable. The monitor in Figure 10 is slightly more deterministic than that of Figure 9 and will generally lead to a less complex reachable region. Note that the selfloops on the violation location have been omitted. Although this affects the behavior of the system, it does so in a way that has no effect on its correctness, assuming we use forward reachability; once a violation has been

\footnotetext{
${ }^{7}$ This assertion is denoted $\forall \square(a \Rightarrow \forall \diamond<\alpha b)$.
} 


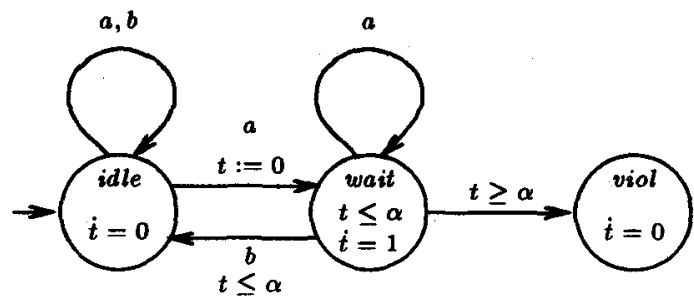

FIGURE 10. Bounded-response monitor automaton - strict bound

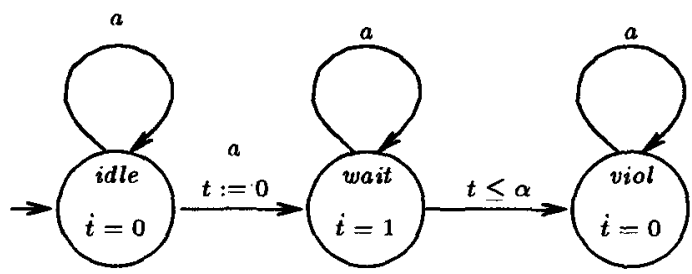

FIGURE 11. Monitor automaton for minimal event-separation time

detected, which additional states are reachable is irrelevant.

Minimal event separation Monitor processes can be built to verify that events occur with some minimal separation time. For example, Figure 11 shows the automaton for verifying that no two instances of the event $a$ occur within $\alpha$ time units of each other.

\section{Installing and Running HYTECH}

\subsection{Installation}

Currently the executable file is available for the Sun4 architecture only. We plan to have versions available for a variety of platforms, including DEC workstations and PC's. Most jobs we have run require less than 20MB, many less than 10MB. However, obviously, the more memory the better.

The version of HYTFCH described here was released in August 1995, and is available through anonymous $\mathrm{ftp}$ via $\mathrm{ftp} . \mathrm{cs}$.cornell.edu in the directory jub/tah/HyTech, and through the World-Wide Web via HrTECH's home page http://www.cs.cornell.edu/Info/People/tah/hytech.html. Download the file hytech.tar.z. It must be uncompressed to hytech.tar, and then expanded using the Unix tar command. The following sequence of commands will produce the directory HyTech. 
uncompress hytech.tar.Z

tar -xf hytech.tar

The HyTech directory contains the subdirectories arc, bin, user guide, examples, and papers, containing the source code, executables, a more comprehensive version of this user guide, examples, and many papers on hybrid automáta, respectively. The main directory also contains the files README and license. Please sign a copy of the license and follow the instructions given on the form. Licensed users will be assured of being informed about new releases of the software. We would also appreciate hearing about your experiences with HYTFCH and the applications you analyze with it.

\subsection{Executing HYTECH}

You must have the files hytech and hytech, exe in your current directory. Assuming your input file is called a.hy, the basic command to run HYTFCH is hytech a.hy. The .hy suffix on the filename may be omitted. Output appears on stdout, so it is usually directed to a file via a command such as hytech a.hy > a.log. HrTFCH creates a temporary file by adding -temp.hy to the source filename, e.g. for the commands above, the file a.hy-temp.hy is temporarily created and then destroyed. Clearly, you should avoid using file names ending in -temp. hy.

Options Available options are displayed by executing НYTFCH with no input file. Options are given in the form - flag_type $\rangle\langle n\rangle$, and must occur before the filename on the command line. The only options so far are for controlling the amount of output generated ( $-\mathrm{p} 0,-\mathrm{p} 1$, and $-\mathrm{p} 2$, where the higher numbers indicate more verbose output), and the format of the output ( $-f 0$ for conjunctions output along a single line, and $-f 1$ for conjuncts listed one per line).

Examples Numerous sample input files and their output logs can be found in the subdirectory examples. Examine these to familiarize yourself with the input description language. Some of them are discussed in the user guide and [HHWT95a].

Bugs, comments, suggestions Please report any bugs or installation and maintenance problems to hytech@cs.cornell.edu. We do not have the resources to provide commercial-level support, but we can probably help you. We also welcome comments and suggestions, since the experience of HYTECH's users will help to improve future versions of the software. 


\section{Hints for the Efficient Use of HyTECH}

This section describes hints on how to make the most of HYTecH's computational power. If HYTECH does not terminate on your input file, and you cannot figure out why, trying these heuristics may well help. Sometimes a slightly modified description will make a tremendous difference. As a general principle, keep your model of the system as simple as possible at first. Once HYTFCH has successfully analyzed the system, slowly add more detail to your model.

Keep the system description small. Generally, the smaller the better, i.e. try to minimize the number of components, locations, and variables. For example, try to model only a small number of the system's components first. Share locations wherever possible, e.g. error locations can often be combined into one. Some locations may be eliminated if they are "intermediate" locations not involved in direct synchronization with other components, and time spent in these locations can be transferred to the immediately adjacent locations.

Encode discrete variables into locations. For a bounded discrete variable, it is generally more efficient to split each location into several locations, one for each value of the variable, than to declare the variable as a real-valued variable. However, the increased efficiency often carries the disadvantage of a less compact description.

Manually compose tightly coupled components. When taking the product of two automata, many product locations are irrelevant since they are unreachable. If two components are tightly coupled with synchronized events, the reachable product automaton can be substantially smaller than the complete product. It may be beneficial to input the reachable product of such automata, instead of their components, since this version of $\mathrm{HYTFCH}$ constructs complete products only.

Keep constants simple. Generally, the lower the $1 \mathrm{~cm}$ :gcd ratio of the constants in the system, the faster the reachability analysis. Indeed, lowering the ratio may be necessary for reachability to terminate. To achieve low lcm:gcd ratios, it is often possible to verify an abstracted system where lower bounds are rounded down to smaller constants, and upper bounds are rounded up.

Model urgent events explicitly. If an event is urgent, model this fact directly where possible by using the ASAP guard. This is more efficient than introducing an auxiliary clock.

Exploit "don't care" information. In many locations of an automaton, not all variable values are relevant. However, reachability analysis will record the exact values of such "don't care" variables. Thus to simplify the reachable region, it is helpful to make these variables completely unknown 
wherever they are irrelevant. This can be achieved by explicitly assigning them into the interval $(-\infty, \infty)$ on all transitions into the appropriate locations. A tempting option is to set them to a particular fixed value while control remains in a given location. However, this strategy is not as beneficial as assigning them into $(-\infty, \infty)$, since there is a nontrivial relationship between them and any other variables as time passes.

Use strong invariants. Sometimes it is helpful to restrict reachability analysis as much as possible through the use of strong invariants. For instance, enforcing implicit invariants can be advantageous, particularly when performing backward reachability analysis. In the gas burner example, backward reachability is required, since forward reachability does not terminate. It would be easy (and natural) to model the system without using the invariants $x \geq 0, y \geq 0$, and $t \geq 0$ for the clock and stopwatch variables. These invariants would play no role in forward analysis. However, backward analysis is nonterminating without these invariants, whereas adding them causes termination in seven iterations.

Use the reachability facility provided. It is optimized for its task and faster than writing your own while loops. It also enables error traces to be generated.

Try forward and backward analysis. It is often not easy to predict which direction will terminate faster.

\section{References}

[ACH93] R. Alur, C. Courcoubetis, and T.A. Henzinger. Computing accumulated delays in real-time systems. In C. Courcoubetis, editor, CAV 93: Computer-aided Verification, Lecture Notes in Computer Science 697, pages 181-193. Springer-Verlag, 1993.

$\left[\mathrm{ACH}^{+}\right.$95] R. Alur, C. Courcoubetis, N. Halbwachs, T.A. Henzinger, P.-H. Ho, X. Nicollin, A. Olivero, J. Sifakis, and S. Yovine. The algorithmic analysis of hybrid systems. Theoretical Computer Science, 138:3-34, 1995.

[ACHH93] R. Alur, C. Courcoubetis, T.A. Henzinger, and P.-H. Ho. Hybrid automata: an algorithmic approach to the specification and verification of hybrid systems. In R.L. Grossman, A. Nerode, A.P. Ravn, and H. Rischel, editors, Hybrid Systems, Lecture Notes in Computer Science 736, pages 209-229. Springer-Verlag, 1993.

[AD94] R. Alur and D.L. Dill. A theory of timed automata. Theoretical Computer Science, 126:183-235, 1994.

[AHH93] R. Alur, T.A. Henzinger, and P.-H. Ho. Automatic symbolic verification of embedded systems. In Proceedings of the 14th Annual Realtime Systems Symposium, pages 2-11. IEEE Computer Society Press, 
1993. Full version available as Technical Report TR-1492, Department of Computer Science, Cornell University, Ithaca, NY 14853, 1995.

[BER.94a] A. Bouajjani, R. Echahed, and R. Robbana. Verification of context-free timed systems using linear hybrid observers. In D.L. Dill, editor, CAV 94: Computer-aided Verification, Lecture Notes in Computer Science, pages 118-131. Springer-Verlag, 1994.

[BER.94b] A. Bouajjani, R. Echahed, and R. Robbana. Verifying invariance properties of timed systems with duration variables. In $\mathrm{H}$. Langmaack, W.-P. de Roever, and J. Vytopil, editors, FTRTFT 94: Formal Techniques in Real-time and Fault-tolerant Systems, Lecture Notes in Computer Science 863, pages 193-210. Springer-Verlag, 1994.

[BR95] A. Bouajjani and R. Robbana. Verifying $\omega$-regular properties for subclasses of linear hybrid systems. In $\mathrm{P}$. Wolper, editor, CAV 95: Computer-aided Verification, Lecture Notes in Computer Science 939, pages 437-450. Springer-Verlag, 1995.

[Cer92] K. Cerāns. Decidability of bisimulation equivalence for parallel timer processes. In G. von Bochmann and D.K. Probst, editors, $C A V$ 92: Computer-aided Verification, Lecture Notes in Computer Science 663, pages 302-315. Springer-Verlag, 1992.

[CH78] P. Cousot and N. Halbwachs. Automatic discovery of linear restraints among variables of a program. In Proceedings of the Fifth Annual Symposium on Principles of Programming Languages. ACM Press, 1978.

[CHR.91] Z. Chaochen, C.A.R. Hoare, and A.P. Ravn. A calculus of durations. Information Processing Letters, 40(5):269-276, 1991.

[DOY94] C. Daws, A. Olivero, and S. Yovine. Verifying ET-LOTOS programs with kRoNos. In Proceedings of Seventh International Conference on Formal Description Techniques, 1994.

[DY95] C. Daws and S. Yovine. Two examples of verification of multirate timed automata with kRONOS. In Proceedings of the 16th Annual Realtime Systems Symposium. IEEE Computer Society Press, 1995.

[Hal93] N. Halbwachs. Delay analysis in synchronous programs. In C. Courcoubetis, editor, CAV 93: Computer-aided Verification, Lecture Notes in Computer Science 697, pages 333-346. Springer-Verlag, 1993.

[Hen92] T.A. Henzinger. Sooner is safer than later. Information Processing Letters, 43:135-141, 1992.

[Hen95] T.A. Henzinger. Hybrid automata with finite bisimulations. In Z. Fülöp and F. Gécseg, editors, ICALP 95: Automata, Languages, and Programming, Lecture Notes in Computer Science 944, pages 324-335. Springer-Verlag, 1995. 
[HH95a] T.A. Henzinger and P.-H. Ho. Algorithmic analysis of nonlinear hybrid systems. In P. Wolper, editor, CAV 95: Computer-aided Verification, Lecture Notes in Computer Science 939, pages 225-238. Springer-Verlag, 1995.

[HH95b] T.A. Henzinger and P.-H. Ho. HYTFCH: The Cornell Hybrid Technology Tool. In A. Nerode, editor, Proceedings of the 1994 Workshop on Hybrid Systems and Autonomous Control, Lecture Notes in Computer Science. Springer-Verlag, 1995.

[HH95c] T.A. Henzinger and P.-H. Ho. A note on abstract-interpretation strategies for hybrid automata. In A. Nerode, editor, Proceedings of the 1994 Workshop on Hybrid Systems and Autonomous Control, Lecture Notes in Computer Science. Springer-Verlag, 1995.

[HHK95] M.R. Henzinger, T.A. Henzinger, and P.W. Kopke. Computing simulations on finite and infinite graphs. In Proceedings of the 36th Annual Symposium on Foundations of Computer Science. IEEE Computer Society Press, 1995.

[HHWT95a] T. A. Henzinger, P.-H. Ho, and H. Wong-Toi. HyTr.CH: the next generation. In Proceedings of the 16th Annual Real-time Systems Symposium. IEEE Computer Society Press, 1995.

[HHWT95b] T. A. Henzinger, P.-H. Ho, and H. Wong-Toi. A user guide to HYTrCH. Technical Report TR-1532, Department of Computer Science, Cornell University, 1995.

[HKPV95] T.A. Henzinger, P.W. Kopke, A. Puri, and P. Varaiya. What's decidable about hybrid automata? In Proceedings of the 27th Annual Symposium on Theory of Computing, pages 373-382. ACM Press, 1995.

[HNSY94] T.A. Henzinger, X. Nicollin, J. Sifakis, and S. Yovine. Symbolic model checking for real-time systems. Information and Computation, 111(2):193-244, 1994.

[Ho95] Pei-Hsin Ho. Automatic Analysis of Hybrid Systems. PhD thesis, Department of Computer Science, Cornell University, 1995.

[HRP94] N. Halbwachs, P. Raymond, and Y.-E. Proy. Verification of linear hybrid systems by means of convex approximation. In B. LeCharlier, editor, SAS 94: Static Analysis Symposium, Lecture Notes in Computer Science 864, pages 223-237. Springer-Verlag, 1994.

[HWT95a] T. A. Henzinger and H. Wong-Toi. Phase portrait approximations of hybrid systems. Submitted, 1995.

[HWT95b] P.-H. Ho and H. Wong-Toi. Automated analysis of an audio control protocol. In P. Wolper, editor, CAV 95: Computer-aided Verification, Lecture Notes in Computer Science 939, pages 381-394. SpringerVerlag, 1995. 
[KPSY93] Y. Kesten, A. Pnueli, J. Sifakis, and S. Yovine. Integration graphs: a class of decidable hybrid systems. In R.L. Grossman, A. Nerode, A.P. Ravn, and H. Rischel, editors, Hybrid Systems, Lecture Notes in Computer Science 736, pages 179-208. Springer-Verlag, 1993.

[Lam87] L. Lamport. A fast mutual exclusion algorithm. ACM Transactions on Computer Systems, 5(1):1-11, 1987.

[LPY95] K. G. Larsen, P. Pettersson, and W. Yi. Compositional and symbolic model-checking of real-time systems. In Proceedings of the 16th Annual Real-time Systems Symposium. IEEE Computer Society Press, 1995.

[LS85] N. Leveson and .I. Stolzy. Analyzing safety and fault tolerance using timed petri nets. In Proceedings of International Joint Conference on Theory and Practice of Software Development, Lecture Notes in Computer Science 186, pages 339-355. Springer-Verlag, 1985.

[MPS95] O. Maler, A. Pnueli, and J. Sifakis. On the synthesis of discrete controllers for timed systems. In E.W. Mayr and C. Puech, editors, STACS 95: Symposium on Theoretical Aspects of Computer Science, Lecture Notes in Computer Science 900, pages 229-242. Springer-Verlag, 1995.

[MV94] J. McManis and P. Varaiya. Suspension automata: a decidable class of hybrid automata. In D.L. Dill, editor, CAV 94: Computer-aided Verification, Lecture Notes in Computer Science 818, pages 105-117. Springer-Verlag, 1994.

[NOSY93] X. Nicollin, A. Olivero, J. Sifakis, and S. Yovine. An approach to the description and analysis of hybrid systems. In R.L. Grossman, A. Nerode, A.P. Ravn, and H. Rischel, editors, Hybrid Systems, Lecture Notes in Computer Science 736, pages 149-178. Springer-Verlag, 1993.

[NSY92] X. Nicollin, J. Sifakis, and S. Yovine. Compiling real-time specifications into extended automata. IEEE Transactions on Software Engineering, SE-18(9):794-804, 1992.

[OSY94] A. Olivero, J. Sifakis, and S. Yovine. Using abstractions for the verification of linear hybrid systems. In D.L. Dill, editor, $C A V$ 94: Computer-aided Verification, Lecture Notes in Computer Science 818, pages 81-94. Springer-Verlag, 1994.

[PV94] A. Puri and P. Varaiya. Decidability of hybrid systems with rectangular differential inclusions. In D.L. Dill, editor, CAV 94: Computeraided Verification, Lecture Notes in Computer Science 818, pages 95104. Springer-Verlag, 1994.

[VW86] M.Y. Vardi and P. Wolper. An automata-theoretic approach to automatic program verification. In Proceedings of the First Annual Symposium on Logic in Computer Science, pages 322-331. IEEE Computer Society Press, 1986. 